\title{
CLNet: Complex Input Lightweight Neural Network designed for Massive MIMO CSI Feedback
}

\author{
Sijie Ji, Mo Li, Fellow, IEEE \\ Nanyang Technological University \\ sijie001@e.ntu.edu.sg, limo@ntu.edu.sg
}

\begin{abstract}
The Massive Multiple Input Multiple Output (MIMO) system is a core technology of the next generation communication. With the growing complexity of CSI, CSI feedback in massive MIMO system has become a bottleneck problem, the traditional compressive sensing based CSI feedback approaches have limited performance. Recently, numerous deep learning based CSI feedback approaches demonstrate their efficiency and potential. However, most existing methods improve accuracy at the cost of computational complexity and the accuracy decreases significantly as the CSI compression rate increases.
\end{abstract}

This paper presents a novel neural network CLNet tailored for CSI feedback problem based on the intrinsic properties of CSI. The experiment result shows that CLNet outperforms the state-of-the-art method by average accuracy improvement of $5.41 \%$ in both outdoor and indoor scenarios with average $24.1 \%$ less computational overhead. Codes are available at GitHub. ${ }^{1}$.

\section{Index Terms}

Massive MIMO, FDD, CSI feedback, deep learning, complex neural network, attention mechanism, lightweight model

\section{INTRODUCTION}

The massive multiple-input multiple-output (MIMO) technology is considered one of the core technologies of the next generation communication system, e.g., 5G. By equipping large number

${ }^{1}$ https://github.com/SIJIEJI/CLNet 
of antennas, base station (BS) can sufficiently utilize spatial diversity to improve channel capacity. Especially, by enabling beamforming, a 5G BS can concentrate signal energy to a specific user equipment (UE) to achieve higher signal-to-noise ratio (SNR), less interference leakage and hence, higher channel capacity. However, beamforming is possibly conducted by the BS only when it has the channel state information (CSI) of the downlink at hand [1].

In frequency division duplexing (FDD) mode, most of contemporary cellular systems operate in [2], the channel reciprocity does not exist. Therefore, the UE would have to explicitly feed back the information of downlink CSI to the BS, and the pilot-aided training overhead grows quadratically with the number of transmitting antennas, which might overturn the benefit of Massive MIMO itself [3]. Thus, CSI compression is needed before the feedback to reduce the overhead.

Traditional compressive sensing (CS) based methods rely heavily on channel sparsity and is limited by their efficiency in iteratively reconstructing the signals. The performance of which is highly dependent on the wireless channel [4], and thus is not a desirable approach considering the diversified use cases of $5 \mathrm{G}$ networks.

The recent rapid development of deep learning (DL) technologies provide another possible solution for efficient CSI feedback for FDD massive MIMO system. Instead of relying on sparsity, the DL approaches utilize the auto-encoder frameworks [5] as an implicit prior constraint for encoding data. The decoder learns a map from the low-dimensional data space to the targeted data distribution by single run to reconstruct the original data, without the labeled data, which naturally overcomes the limits of CS-based approaches in channel sparsity and operation efficiency.

The first DL-based method, CsiNet [6], explored and demonstrated the efficiency of deep learning in CSI feedback. The proposed CsiNet based on convolutional neural network (CNN) and designed two sequential RefineNet in the decoder to refine the reconstruction accuracy. CsiNet significantly outperforms the traditional CS-based methods (LASSO, BM3D-AMP and TVAL3) under various compression rates.

Based on CsiNet, most of the subsequent DL-based methods utilize more powerful DL building blocks to achieve better performance with the sacrifice of computational overhead. CsiNetLSTM [7] and Attension-CSI [8] introduced LSTM that significantly increases the computational overhead. CsiNet+ [9] comprehensively surveyed recent DL-based methods and proposed a parallel multiple-rate compression framework. DS-NLCsiNet [10] adopts non-local block. The computational overhead of CsiNet+ and DS-NLCsiNet are approximately x6 and x 2.5 higher than 
the original CsiNet respectively [11]. Recently, some methods start to reduce the complexity, for example, JCNet [12] and BcsiNet [13], however, their performance also degraded. So far, only CRNet [14] outperforms CsiNet without increasing the computational complexity.

Different from previous works, this work based on the inherent characteristic of CSI data, considering the limited computation resource and limited storage at UE side, and design a tailored lightweight DL framework, CLNet, for CSI feedback problem. Eventually, CLNet outperforms CRNet with $5.41 \%$ higher accuracy and $24.1 \%$ less complexity on average. The main contributions are summarized as follows:

- Signals and CSI are represented in complex envelopes, but at present, the majority of building blocks for DL models are based on real-valued operations and representations. CLNet proposes a simple yet effective way to organic integrate the real and imaginary parts into the real-valued neural network models.

- Different signal paths are clustered in the angular-delay domain, thus CLNet introduces two attention mechanisms to let the DL model focus on the more informative part.

- CQNet is a lightweight network and reduces the hardware-depends operations, such as exponent calculation.

The rest of this paper is organized as follows. Section II introduces the system model and preliminary, including the channel model and CSI feedback process. Section III presents the detailed design of CLNet. Section IV provides experimental details and evaluates the performance of CLNet. Section V concludes the paper.

\section{System MOdel AND PRELIMINARY}

Consider a single cell FDD system using massive MIMO with $N_{t}$ antennas at BS, where $N_{t}$ $\gg 1$ and $N_{r}$ antennas at UE side ( $N_{r}$ equals to 1 for simplicity). The received signal $y \in \mathbb{C}^{N_{c} \times 1}$ can be expressed as

$$
y=\boldsymbol{A} x+z
$$

where $N_{c}$ indicates the number of subcarriers, $x \in \mathbb{C}^{N_{c} \times 1}$ indicates the transmitted symbols, and $z \in \mathbb{C}^{N_{c} \times 1}$ is the complex additive Gaussian noise. A can be expressed as $\operatorname{diag}\left(h_{1}^{H} p_{1}, \cdots, h_{N_{c}}^{H} p_{N_{c}}\right)$, where $h_{i} \in \mathbb{C}^{N_{t} \times 1}$ and $p_{i} \in \mathbb{C}^{N_{t} \times 1}, i \in\left\{1, \cdots, N_{c}\right\}$ represent downlink channel coefficients and beamforming precoding vector for subcarrier $i$, respectively.

In order to derive the beamforming precoding vector $p_{i}$, the $\mathrm{BS}$ needs the knowledge of corresponding channel coefficient $h_{i}$, which is fed back by the UE. Suppose that the downlink 
channel matrix is $\boldsymbol{H}=\left[h_{1} \cdots h_{N_{c}}\right]^{H}$ which contains $N_{c} N_{t}$ elements. The number of parameters that need to be fed back is $2 N_{c} N_{t}$, including the real and imaginary parts of the CSI, which is proportional to the number of antennas.

Because the channel matrix $\boldsymbol{H}$ is often sparse in the angular-delay domain. By 2D discrete Fourier transform (DFT), the original form of spatial-frequency domain CSI can be converted into angular-delay domain, such that

$$
\boldsymbol{H}^{\prime}=\boldsymbol{F}_{c} \boldsymbol{H F}_{t}^{H}
$$

where $\boldsymbol{F}_{c}$ and $\boldsymbol{F}_{t}$ are the DFT matrices with dimension $N_{c} \times N_{c}$ and $N_{t} \times N_{t}$, respectively. For the angular-delay domain channel matrix $\boldsymbol{H}^{\prime}$, every element corresponds to a certain path delay with a certain angle of arrival (AoA). In $\boldsymbol{H}^{\prime}$, only the first $N_{a}$ rows contain useful information, while the rest rows represent the paths with larger propagation delays are made up of near-zero values, can be omitted without much information loss. Let $\boldsymbol{H}_{a}$ denote the informative rows of $\boldsymbol{H}^{\prime}$.

$\boldsymbol{H}_{a}$ is input into UE's encoder to produce the codeword $\boldsymbol{v}$ according to a given compression ratio $\eta$ such that

$$
\boldsymbol{v}=f_{\mathcal{E}}\left(\boldsymbol{H}_{a}, \Theta_{\mathcal{E}}\right)
$$

where $f_{\mathcal{E}}$ denotes the encoding process and $\Theta_{\mathcal{E}}$ represents a set of parameters of the encoder.

Once the BS receives the codeword $v$, the decoder is used to reconstruct the channel by

$$
\hat{\boldsymbol{H}}_{a}=f_{\mathcal{D}}\left(\boldsymbol{v}, \Theta_{\mathcal{D}}\right)
$$

where $f_{\mathcal{D}}$ denotes the decoding process and $\Theta_{\mathcal{D}}$ represents a set of parameters of the decoder. Therefore, the entire feedback process can be expressed as

$$
\hat{\boldsymbol{H}}_{a}=f_{\mathcal{D}}\left(f_{\mathcal{E}}\left(\boldsymbol{H}_{a}, \Theta_{\mathcal{E}}\right), \Theta_{\mathcal{D}}\right)
$$

The goal of CLNet is to minimize the difference between the original $\boldsymbol{H}_{a}$ and the reconstructed $\hat{\boldsymbol{H}}_{a}$, which can be expressed formally as finding the parameter sets of encoder and decoder satisfying

$$
\left(\hat{\Theta}_{\mathcal{E}}, \hat{\Theta}_{\mathcal{D}}\right)=\underset{\Theta_{\mathcal{E}}, \Theta_{\mathcal{D}}}{\arg \min }\left\|\boldsymbol{H}_{a}-f_{\mathcal{D}}\left(f_{\mathcal{E}}\left(\boldsymbol{H}_{a}, \Theta_{\mathcal{E}}\right), \Theta_{\mathcal{D}}\right)\right\|_{2}^{2}
$$




\section{CLNet DESIGN}

This section presents the design of the CLNet and its key components. Figure 1 depicts the overall architecture of CLNet, traditional convolution blocks are omitted for simplicity. Overall, CLNet is an encoder-decoder framework with four main building blocks that are tailored to the CSI feedback problem.

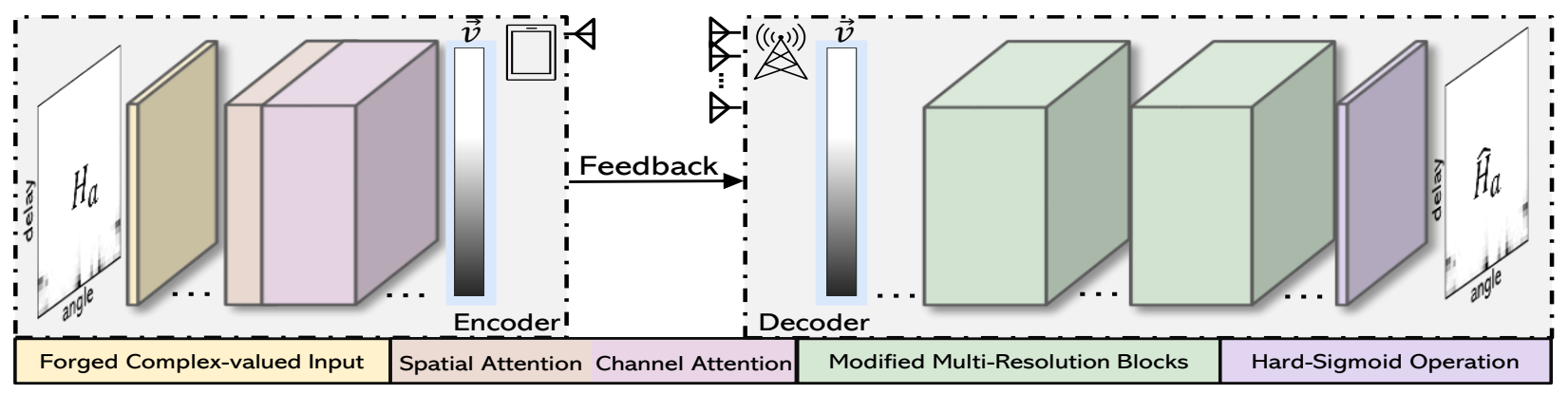

Fig. 1: The encoder and decoder architecture of CLNet.

The performance of the CSI feedback scheme highly depends on the compression part, the encoder. The less information loss of the compression, the higher the decompression accuracy can be obtained. Due to the limited computing power and storage space of UE, deepening the encoder network design is not practical. Therefore, CLNet leverages the physical characteristics of CSI to achieve a lightweight yet informative encoder by two tailored blocks.

First, CSI is the channel frequency response with complex-valued that depicts channel coefficients of different signal paths. The previous DL-based CSI feedback methods, which treat the real and imaginary parts of the CSI separately. Instead, the input CSI in CLNet first goes through the forged complex-valued input layer that embeds the real and imaginary parts together to preserve the physical information of the CSI (Section III-A). Second, different signal paths have different resolutions of cluster effect in the angular-delay domain, which corresponding to different angles of arrival and different path delays. Thus, we introduce two types of attention blocks that force the neural network focus on those clusters with paths information and suppress the unnecessary parts (Section III-B).

Since the encoder becomes more powerful, the decoder can be correspondingly more lightweight, thus CLNet modifies the CRBlocks [14] in decoder by reducing the filter size from $1 \times 9$ to $1 \times 3$. To further reduce the computational cost, CLNet adopts the hard-Sigmoid activation fuction 
which is more hardware friendly than the conventional Sigmoid activation function (Section IIIC).

\section{A. Forged Complex-valued Input}

A typical deep learning neural network is designed based on real-valued inputs, operations, and representations. How to best cope with complex-valued inputs is yet an open question in the machine learning community [15]. CSI is complex-valued channel coefficients, all of the existing DL-based methods for wireless communication or wireless sensing systems separate the real and imaginary parts of the complex-valued signals, treat them as two independent channels of an image as input, and perform mixed convolutions around the real and imaginary parts of multiple signals, which may destroy the original physical property of each complex-valued channel coefficient. Specifically, as Figure 2 (b) depicts, a conventional $3 \times 3$ kernel size entangles the real and imaginary parts of neighboring elements in $\boldsymbol{H}_{a}$, and as a result, the 9 complex values are interpolated as one synthesized value. In such way, the real and imaginary parts of the same complex-valued signal are decoupled during the convolution process, thus losing the original physical information carried by the channel matrix.

To tackle such an issue, CLNet devises a specific input layer, which utilizes a $1 \times 1$ point-wise convolution to couple the real and imaginary parts of the same channel coefficient. The forged complex-valued input layer employs multiple $1 \times 1$ convolutional filters to encode the real and imaginary parts of each complex-valued element in $\boldsymbol{H}_{a}$ with respective learnable weights.

Mathematically, $\boldsymbol{F}_{t r}: \boldsymbol{H}_{a} \rightarrow \mathcal{I}$ is a convolutional transformation. Here, $\boldsymbol{H}_{a} \in \mathbb{R}^{N_{a} \times N_{a} \times 2}$ is a 3D tensor, extended from its $2 \mathrm{D}$ version by including an additional dimension to separately express the real and imaginary parts, and $\mathcal{I} \in \mathbb{R}^{N_{a} \times N_{a} \times C}$, where $C$ indicates the number of convolutional filters applied to learn different weighted representations. Let $\boldsymbol{F}=\left[\boldsymbol{f}_{1}, \boldsymbol{f}_{2}, \ldots, \boldsymbol{f}_{C}\right]$ denote the learned set of filter kernels, where $\boldsymbol{f}_{c}$ refers to the learnable parameter of the $c$-th filter. The output of $\boldsymbol{F}_{t r}$ is $\mathcal{I}=\left[\boldsymbol{i}_{1}, \boldsymbol{i}_{2}, \ldots, \boldsymbol{i}_{C}\right], \boldsymbol{i}_{c} \in \mathbb{R}^{N_{a} \times N_{a}}$, where

$$
\boldsymbol{i}_{c}[m, n]=\boldsymbol{f}_{c} * \boldsymbol{H}_{a}=\sum_{i=1}^{m} \sum_{j=1}^{n} \sum_{k=1}^{2} \boldsymbol{f}_{c}[i, j] \boldsymbol{H}_{a}^{k}[m-i, n-j]
$$

Here $*$ denotes convolution, and $m, n \in\left[0, N_{a}\right)$. For simplicity, bias terms are omitted. Since the output is produced by a summation of the two channels, the dependency between real and imaginary parts is implicitly embedded in $\boldsymbol{i}_{c}$. Based on the trade-off between accuracy and model size, CLNet adopts $C=32$ as learnable filters. 
Figure 2 (a) illustrates the design of the complex-valued input layer, $\boldsymbol{F}_{t r}$, whose output will be directed to the attention mechanism $\boldsymbol{F}_{s e}$ (to be detailed in next section).

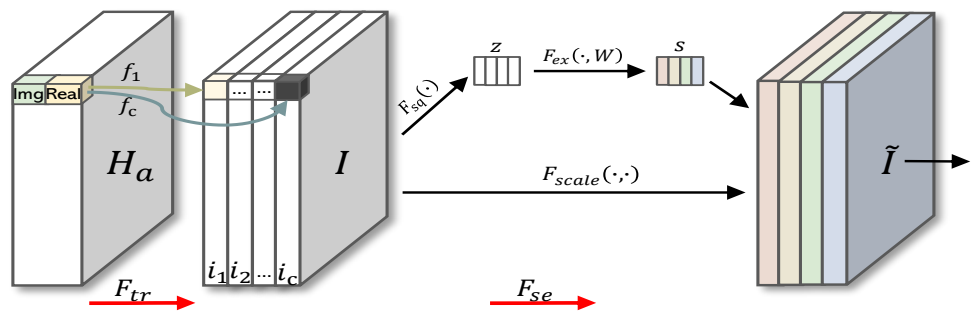

(a) CQNet forged complex-valued input and attention design

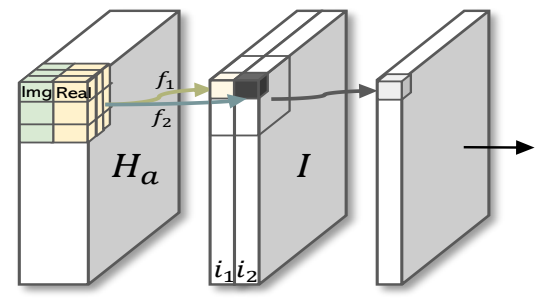

(b) Conventional convolution design

Fig. 2: (a) The forged complex-valued input operation $F_{t r}$ couples the real and imaginary parts of the complex-valued channel matrix and after that the channel-wise attention $F_{s e}$ is applied to strengthen learning of significant channel coefficients. (b) Conventional convolution entangles the real and imaginary parts with neighboring elements' real parts and imaginary parts.

\section{B. Attention Mechanism for Informative Encoder}

Attention mechanism biases the allocation of available computational resources to the most informative components of a signal that increases the representativeness of the neural network. CLNet imposes two different attention mechanisms for different purposes, i.e., the channel-wise attention block which aims at enhancing the effectiveness of complex-valued input layer, and the spatial-wise attention block which aims at making use of the cluster effect in the angular-delay domain.

1) Channel-Wise Attention: To keep the whole information of the complex annotated $\boldsymbol{H}_{a}$, CLNet devises a forged complex-valued input layer. The output of the input layer $\mathcal{I}$, essentially, is a weighted representation of the original $\boldsymbol{H}_{a}$. Specifically, $\mathcal{I} \in \mathbb{R}^{N_{a} \times N_{a} \times C}$, where the number of channel $C$ corresponds to the learned different weights of $\boldsymbol{H}_{a}$, among which, some may be more important than others. Based on this, CLNet introduces the SE block [16], to assist the neural network model with the relationship of the weights to focus on the important features and suppress the unnecessary ones. A diagram of the SE block is shown in Figure 2 (a) with annotation $\boldsymbol{F}_{s e}$. 
The output $\mathcal{I}$ first goes through $\boldsymbol{F}_{s q}$ transformation by global average pooling to obtain channelwise statistics descriptor $z \in \mathbb{R}^{C}$,

$$
z_{c}=\boldsymbol{F}_{s q}\left(\boldsymbol{i}_{c}\right)=\frac{1}{N_{a}^{2}} \sum_{i=1}^{N_{a}} \sum_{j=1}^{N_{a}} \boldsymbol{i}_{c}(i, j), \text { s.t. } c \in\{1, \cdots, C\}
$$

Here, $\boldsymbol{F}_{s q}$ expands the neural network receptive field to the whole angular-delay domain to obtain the global statistical information, compensating for the shortcoming of the insufficient local receptive field of $1 \times 1$ convolution that used in the forged complex-valued input layer.

After that, the channel descriptor $\boldsymbol{z}$ goes through $\boldsymbol{F}_{\text {ex }}$ transformation, i.e., a gated layer with sigmoid activation to learn the nonlinear interaction as well as the non-mutually-exclusive relationship between channels, such that

$$
\mathbf{s}=\boldsymbol{F}_{e x}(\boldsymbol{z}, \boldsymbol{W})=\sigma(g(\boldsymbol{z}, \boldsymbol{W}))=\sigma\left(\boldsymbol{W}_{2} \delta\left(\boldsymbol{W}_{1} \boldsymbol{z}\right)\right)
$$

where $\delta$ is the ReLU function, $\boldsymbol{W}_{1} \in \mathbb{R}^{\frac{C}{2} \times C}$ and $\boldsymbol{W}_{2} \in \mathbb{R}^{C \times \frac{C}{2}}$. $\boldsymbol{F}_{\text {ex }}$ further explicitly models the inter-channel dependencies based on $z$ and obtain the calibrated $\mathrm{s}$, which is the attention vector that summarizes all the characteristics of channel $C$, including intra-channel and interchannel dependencies. Before being fed into the next layer, each channel of $\mathcal{I}$ is scaled by the corresponding attention value, such that

$$
\tilde{\mathcal{I}}_{:,, i}=\boldsymbol{F}_{\text {scale }}(\mathbf{s}, \mathcal{I})=\mathbf{s}_{i} \mathcal{I}_{:,:, i}, \text { s.t. } i \in\{1,2, \cdots, C\}
$$

The channel-wise attention mechanism intrinsically captures dynamics based on the complexvalued input $\boldsymbol{H}_{a}$ by learning to weigh the importance of each channel in $\mathcal{I}$, boosts the feature discriminability, and generates a more informative $\tilde{\mathcal{I}}$.

2) Spatial-Wise Attention: Spatial-wise attention focuses on learning the places of the more informative parts across the spatial domain. Specifically, after being converted to the angular-delay domain, the channel coefficients exhibit the effect of clusters corresponding to the distinguishable paths that arrive with specific delays and AoAs. In order to pay more attention to such clusters, CLNet employs a CBAM block [17] to distinguish them with weights in the spatial domain as Figure 3 illustrates.

First, two pooling operations, i.e., average-pooling and max-pooling, are adopted across the input $\boldsymbol{F}_{i}$ 's channel $C$ to generate two $2 \mathrm{D}$ feature maps, $\boldsymbol{F}_{\text {avg }} \in \mathbb{R}^{N_{a} \times N_{a} \times 1}$ and $\boldsymbol{F}_{\max } \in \mathbb{R}^{N_{a} \times N_{a} \times 1}$, respectively. CLNet concatenates the two feature maps to generate a compressed spatial feature descriptor $\boldsymbol{F}_{\text {dsc }} \in \mathbb{R}^{N_{a} \times N_{a} \times 2}$, and convolves it with a standard convolution layer to produce a $2 \mathrm{D}$ 


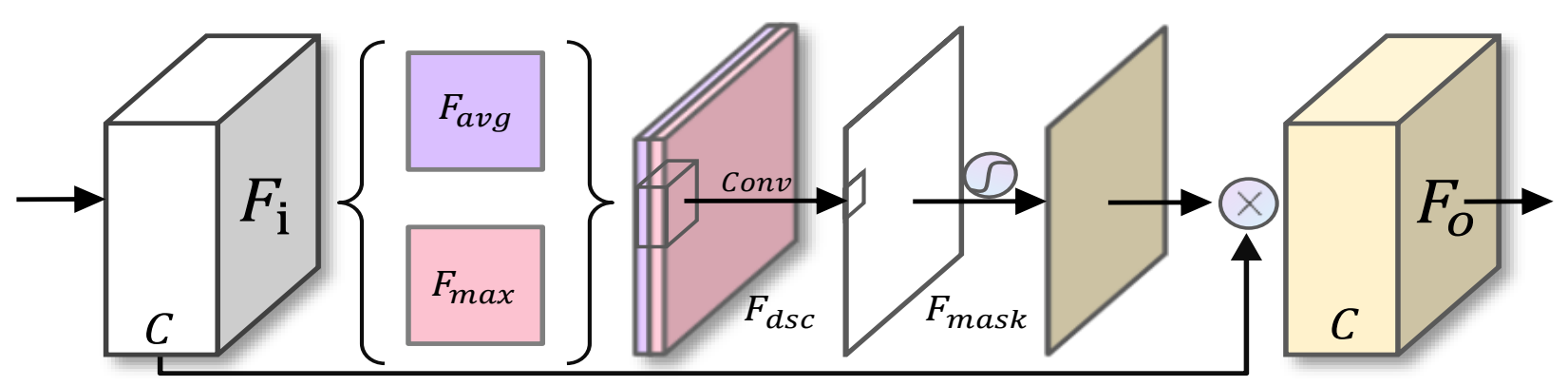

Fig. 3: Based on the cluster effect in the angular-delay domain, spatial-wise attention uses the generated spatial statistical descriptors as the basis for assigning weights, forcing the network to focus more on the distinguishable propagation paths.

spatial attention mask $\boldsymbol{F}_{\text {mask }} \in \mathbb{R}^{N_{a} \times N_{a} \times 1}$. The mask is activated by Sigmoid and then multiplied with the original feature maps $\boldsymbol{F}_{i}$ to obtain $\boldsymbol{F}_{o}$ with spatial-wise attention.

$$
\begin{aligned}
\boldsymbol{F}_{o} & =\operatorname{CBAM}\left(\boldsymbol{F}_{i}\right) \\
& =\boldsymbol{F}_{i}\left(\sigma\left(\boldsymbol{f}_{c}\left(\left[\operatorname{Avg} \operatorname{Pool}\left(\boldsymbol{F}_{i}\right) ; \operatorname{Max} \operatorname{Pool}\left(\boldsymbol{F}_{i}\right)\right]\right)\right)\right) \\
& =\boldsymbol{F}_{i}\left(\sigma\left(\boldsymbol{f}_{c}\left(\left[\boldsymbol{F}_{\text {avg }} ; \boldsymbol{F}_{\text {max }}\right]\right)\right)\right)
\end{aligned}
$$

With spatial-wise attention, CLNet focuses the neural network on the more informative signal propagation paths in the angular-delay domain.

\section{Reduction of Computational Cost}

The often-used Sigmoid activation function contains exponential operation:

$$
\sigma(x)=\frac{1}{1+e^{-x}}=\frac{e^{x}}{e^{x}+1} .
$$

In order to reduce time cost in the computation, CLNet uses the hard version of Sigmoid, its piece-wise linear analogy function, denoted as $h \sigma$ to replace the Sigmoid function [18],

$$
h \sigma(x)=\frac{\min (\max (x+3,0), 6)}{6}
$$

Figure 4 compares the excitation curves of the hard-Sigmoid and Sigmoid functions. The hardSigmoid induces no discernible degradation in the accuracy but benefits from its computational advantage of entailing no exponential calculations. In practice, the hard-Sigmoid can fit in most software and hardware frameworks and can mitigate the potential numerical quantization loss introduced by different hardware. 


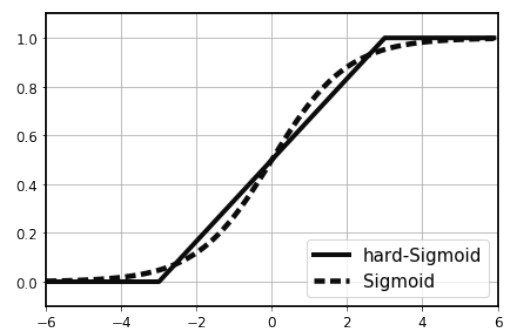

Fig. 4: Comparison between Sigmoid and hard-Sigmoid functions.

\section{EVALUATION}

This section presents the detailed experiment setting and the comparison with the state-of-theart DL-based CSI feedback approach, CRNet, in terms of accuracy and computational overhead.

1) Data Generation: To ensure a fair performance comparison, we use the same dataset as provided in the first work of deep learning-based Massive MIMO CSI feedback in [6], which is also used in later studies on this problem [9], [14], [7]. The generated CSI matrices are converted to angular-delay domain $\boldsymbol{H}_{a} \in \mathbb{R}^{32 \times 32 \times 2}$ by $2 \mathrm{D}$-DFT. The total 150,000 independently generated CSI are split into three parts, i.e., 100,000 for training, 30,000 for validation, and 20,000 for testing, respectively.

2) Training Scheme and Evaluation Metric: As the comparison scheme, we use the startof-the-art method CRNet [14], which significantly outperforms other CSI feedback work. To evaluate the performance, we measure the normalized mean square error (NMSE) between the original $\boldsymbol{H}_{a}$ and the reconstructed $\hat{\boldsymbol{H}}_{a}$ :

$$
\mathrm{NMSE}=\mathrm{E}\left\{\left\|\boldsymbol{H}_{a}-\hat{\boldsymbol{H}}_{a}\right\|_{2}^{2} /\left\|\boldsymbol{H}_{a}\right\|_{2}^{2}\right\}
$$

The model was trained with the batch size of 200 and 8 workers on a single NVIDIA 2080Ti GPU. The epoch is set to 1000 , as recommended in previous work [14], [9]. To further ensure the fairness, we fix the random seed of the computer ${ }^{2}$.

Figure 5 shows the overall performance of CLNet as compared with CRNet, with the same hardware condition and training scheme. In indoor scenarios, CLNet obtains an average performance increase of $6.61 \%$, with the most increase of $21.00 \%$ at the compression ratio of $\eta=1 / 4$. In outdoor scenarios, the average improvement on NMSE is $4.21 \%$, the most increase $(10.44 \%)$

\footnotetext{
${ }^{2}$ We reproduce CRNet follow the open source code: https://github.com/Kylin9511/CRNet the higher performance they reported in the paper are from training with 2500 epoch.
} 

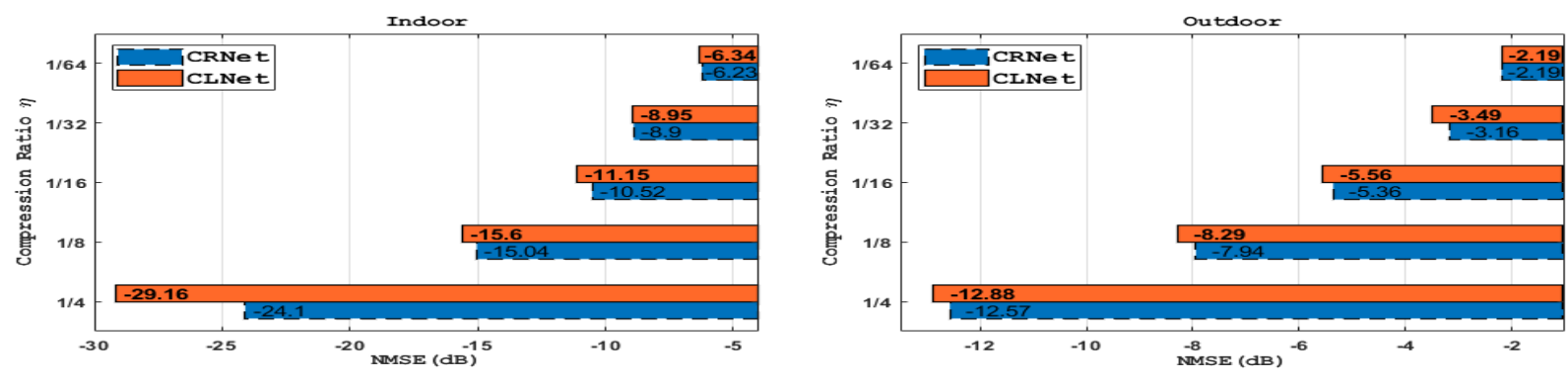

Fig. 5: Normalized Mean Square Error(dB) Comparison between CRNet and CLNet.

occurs at the compression ratio of $\eta=1 / 32$. The result shows that CLNet consistently outperforms CRNet for all compression ratios in both indoor and outdoor scenarios with $5.41 \%$ overall average improvement on NMSE. In addition, both CLNet and CRNet have lower accuracy in outdoor scenarios, which is probably caused by the data processing. Due to the long propagation distance, the propagation loss and the path delay in outdoor scenarios are larger, leading to part of the information being discarded when calculating $\boldsymbol{H}_{a}$.

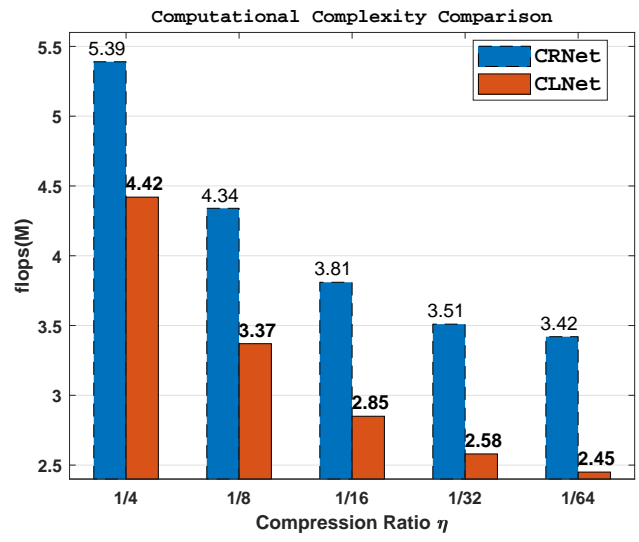

Fig. 6: the Number of flops $\left(\times 10^{6}\right)$ of CRNet and CLNet.

In addition to the accuracy improvement, at the same time, the computational cost in flops (floating-point operations per second) of the two models are compared ${ }^{3}$. As Figure 6 indicates, CLNet significantly reduces the computational complexity with $24.1 \%$ fewer flops on average,

\footnotetext{
${ }^{3}$ Note that the CRNet paper reported flops is corrected by [13], this result is caculated by pytorch-OpCounter: https://github.com/Lyken17/pytorch-OpCounter.
} 
which indicates that CLNet achieves a higher accuracy with less computational complexity. The number of flops of CLNet is $18.00 \%, 22.35 \%, 25.20 \%, 26.50 \%, 28.36 \%$ less than CRNet at the compression ratio $\eta$ of $1 / 4,1 / 8,1 / 16,1 / 32$ and $1 / 64$, respectively. As the compression rate increases, the computational complexity degrades more.

\section{CONCLUSION}

This paper studies the CSI feedback problem for massive MIMO under frequency division duplexing mode, which is the key technology of 5G communication systems. Based on the understanding of the physical properties of the CSI data, a novel customized deep learning framework, CLNet, is proposed. CLNet enhances the pseudo-complex input layer with attention mechanisms to obtain a distilled informative encoder. The modified decoder with smaller filter size utilizes hard-Sigmoid function to eliminate the exponential calculations. The overall performance of CLNet has $5.41 \%$ higher accuracy than the state-of-the-art CRNet with $24.1 \%$ less computation overhead. Since the computational resource of UE is limited, the lightweight CLNet is more suitable for practical deployment.

\section{REFERENCES}

[1] T. L. Marzetta, "Noncooperative cellular wireless with unlimited numbers of base station antennas," IEEE transactions on wireless communications, vol. 9, no. 11, pp. 3590-3600, 2010.

[2] T. L. Marzetta, G. Caire, M. Debbah, I. Chih-Lin, and S. K. Mohammed, "Special issue on massive mimo," Journal of communications and networks, vol. 15, no. 4, pp. 333-337, 2013.

[3] L. Lu, G. Y. Li, A. L. Swindlehurst, A. Ashikhmin, and R. Zhang, "An overview of massive mimo: Benefits and challenges," IEEE journal of selected topics in signal processing, vol. 8, no. 5, pp. 742-758, 2014.

[4] P. Kyritsi, D. C. Cox, R. A. Valenzuela, and P. W. Wolniansky, "Correlation analysis based on mimo channel measurements in an indoor environment," IEEE Journal on Selected areas in communications, vol. 21, no. 5, pp. 713-720, 2003.

[5] G. E. Hinton and R. R. Salakhutdinov, "Reducing the dimensionality of data with neural networks," science, vol. 313 , no. 5786, pp. 504-507, 2006.

[6] C.-K. Wen, W.-T. Shih, and S. Jin, "Deep learning for massive mimo csi feedback," IEEE Wireless Communications Letters, vol. 7, no. 5, pp. 748-751, 2018.

[7] T. Wang, C.-K. Wen, S. Jin, and G. Y. Li, "Deep learning-based csi feedback approach for time-varying massive mimo channels," IEEE Wireless Communications Letters, vol. 8, no. 2, pp. 416-419, 2018.

[8] Q. Cai, C. Dong, and K. Niu, “Attention model for massive mimo csi compression feedback and recovery," in 2019 IEEE Wireless Communications and Networking Conference (WCNC). IEEE, 2019, pp. 1-5.

[9] J. Guo, C.-K. Wen, S. Jin, and G. Y. Li, "Convolutional neural network-based multiple-rate compressive sensing for massive mimo csi feedback: Design, simulation, and analysis," IEEE Transactions on Wireless Communications, vol. 19, no. 4, pp. 2827-2840, 2020. 
[10] X. Yu, X. Li, H. Wu, and Y. Bai, "Ds-nlcsinet: Exploiting non-local neural networks for massive mimo csi feedback," IEEE Communications Letters, vol. 24, no. 12, pp. 2790-2794, 2020.

[11] Z. Lu, X. Zhang, H. He, J. Wang, and J. Song, "Binarized aggregated network with quantization: Flexible deep learning deployment for csi feedback in massive mimo system," arXiv preprint arXiv:2105.00354, 2021.

[12] C. Lu, W. Xu, S. Jin, and K. Wang, "Bit-level optimized neural network for multi-antenna channel quantization,” IEEE Wireless Communications Letters, vol. 9, no. 1, pp. 87-90, 2019.

[13] Z. Lu, J. Wang, and J. Song, "Binary neural network aided csi feedback in massive mimo system," IEEE Wireless Communications Letters, 2021.

[14] — - "Multi-resolution csi feedback with deep learning in massive mimo system," pp. 1-6, 2020.

[15] C. Trabelsi, O. Bilaniuk, Y. Zhang, D. Serdyuk, S. Subramanian, J. F. Santos, S. Mehri, N. Rostamzadeh, Y. Bengio, and C. J. Pal, "Deep complex networks," arXiv preprint arXiv:1705.09792, 2017.

[16] J. Hu, L. Shen, and G. Sun, "Squeeze-and-excitation networks," in Proceedings of the IEEE conference on computer vision and pattern recognition, 2018, pp. 7132-7141.

[17] S. Woo, J. Park, J.-Y. Lee, and I. So Kweon, "Cbam: Convolutional block attention module," in Proceedings of the European conference on computer vision (ECCV), 2018, pp. 3-19.

[18] A. Howard, M. Sandler, G. Chu, L.-C. Chen, B. Chen, M. Tan, W. Wang, Y. Zhu, R. Pang, V. Vasudevan et al., "Searching for mobilenetv3," in Proceedings of the IEEE International Conference on Computer Vision, 2019, pp. 1314-1324. 